\title{
Design and Experimental Evaluation of a Human Skill-Based PID Controller
}

\author{
Yuntao Liao \\ Graduate School of Engineering, Hiroshima University, \\ Hiroshima Japan \\ Toru Yamamoto \\ Faculty of Engineering, Hiroshima University \\ Hiroshima Japan \\ E-mail:liao-yuntao@hiroshima-u.ac.jp,yama@hiroshima-u.ac.jp \\ www.hirosima-u.ac.jp
}

\begin{abstract}
Nowadays less and less people are willing to do hard works. Which makes that the controller based on human skill is needed. Cerebellar model articulation controller (CMAC) is a kind of neural networks (NNs) that can easily solve problems of nonlinear system. Compared with other NNs the advantage of CMAC is that it takes shorter learning time. Moreover because of the widely used of PID controller a human skill-based PID controller using CMACs has been proposed in this paper.
\end{abstract}

Keywords: Cerebellar model articulation controller, Neural network, Human skill, PID controller.

\section{Introduction}

In the last decades, the automatic controller has been widely used in many fields. At the same time the technique of human skill is still playing an important role in control field. However due to the change of modern thoughts the skilled workers become less and less. As the result these specific skills will not be taken to the next generations. Therefore, to develop human skill based controller is necessary.

Human skill is a kind of nonlinear system. In order to learn the nonlinearity of such systems, it is effective that some neural networks (NNs) are utilized. So far many NNs have been proposed and parts of them have been successfully used to design controllers ${ }^{1-4}$. However there is a problem that the conventional NNs need large learning time to adjust weights of neurons, which limits the application of it. The appearance of cerebellar model articulation controller (CAMC) that was proposed at 1975 by Albus partly solves this problem. CMAC has simple structure and weights of CMAC also need to be adjusted. This kind of adjustment has generalization ability which leads CMAC takes less time for computation and some CMAC controllers have already been proposed ${ }^{5}$. Due to the above reasons by using CMAC to learn the nonlinearity of human skill is feasible.

Furthermore PID controller has been widely used in various control systems. Also the experienced human experts have been inevitably required in tuning PID gains. For the above proposal a human-skill based PID controller using CAMCs have been proposed.

\section{Design of a Human Skill-Based Controller}

\section{1. $C M A C$}

The following figure is a sample of simplified 2dimensional input CMAC model to explain the structure of CMAC. 


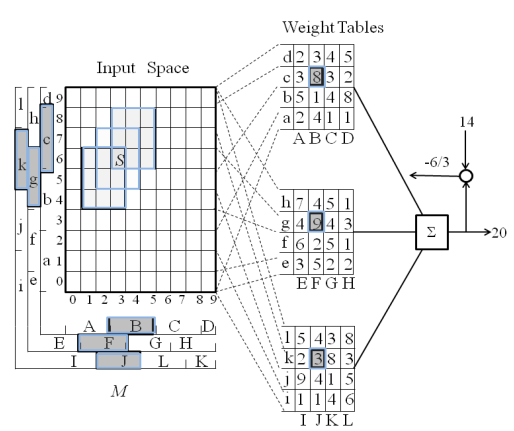

Fig.1. 2-dimensional input CMAC model

In Fig. 1, it is a CMAC with 3 weight tables and 4 labels. The set of input signals $\mathrm{S}(3,6)$ is mapped to B, F, J and $\mathrm{c}, \mathrm{g}, \mathrm{k}$ as set of labels $\mathrm{M}$. Then, from each weight table 8,9 and 3 are selected and sum of them is output 20. Assume that the desired output value is 14 , there will be a $-6 / 3$ added to all referred weights in the tables because of the difference between obtained value and wished value is -6 and the number of selected weight tables is 3 . As explained in the example, CMAC usually works as follows: First the set of input signals $\mathrm{S}$ is mapped to the set of labels M. Then, according to the set of tables weights will be selected from each table. At last, output signals will be performed as the sum of the weights. From the explanation, when CMAC updating weights for one input signal the weights for the nearest input signals are also being updated, this kind of ability makes CMAC can obtain approximately desired value in shorter learning time than conventional NNs.

\subsection{Controller design}

In this paper, the following PID structure is considered:

$$
\begin{gathered}
u(t)=u(t-1)+K_{p}(y(t-1)-y(t))+K_{i} e(t) \\
+K_{d}(2 y(t-1)-y(t)-y(t-2))
\end{gathered}
$$

where $\mathrm{Kp}, \mathrm{Ki}$ and $\mathrm{Kd}$ denote the proportional gain, the integral gain and the differential gain. Moreover $r(t)$ denotes the target value and $\mathrm{e}(\mathrm{t})$ means the control error signal. The block diagram that shows the process how proposed method works is Fig.2.

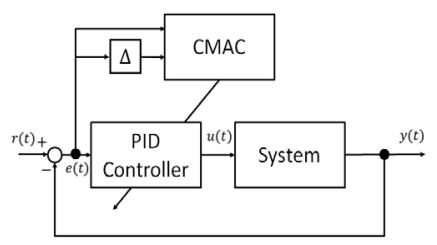

Fig.2. Block diagram of proposed method
In figure human skill data has already been learned by the CMAC. PID gains that tuned by these CMACs are used to control the system. The CMAC here sets $e(t)$ and $\Delta \mathrm{e}(\mathrm{t})$ as the input space to calculate weights. $\Delta \mathrm{e}(\mathrm{t})$ is the signal defined as:

$$
\Delta e(t):=e(t)-e(t-1)
$$

PID parameters are tuned by CMACs according to the following equations:

$$
\begin{aligned}
& K_{p}=\sum_{h=1}^{K} W_{p, h}(t) \\
& K_{i}=\sum_{h=1}^{K} W_{i, h}(t) \\
& K_{d}=\sum_{h=1}^{K} W_{d, h}(t)
\end{aligned}
$$

Where $\mathrm{h}=1,2 \ldots, \mathrm{K}$ and $\mathrm{K}$ denotes the total number of the selected weights in CMAC. From above, the human skill based controller is designed through PID gains. And the performance of the controller is strongly depended on these PID gains.

\subsection{Learning Process}

Then the process of how CMACs learn human-skill data is shown in Fig.3.

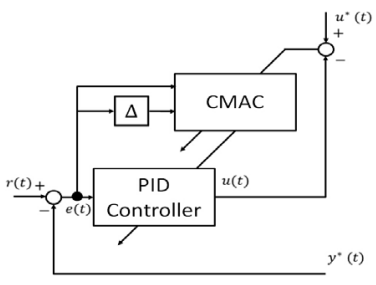

Fig.3. Block diagram of learning process

From Fig. 3 there are two signals that attached asterisk (*) which means they are human-skill data. Humanskilled signals are set as the teacher signals. The learned result $\mathrm{u}(\mathrm{t})$ approaches teacher signal $\mathrm{u}^{*}(\mathrm{t})$ by updating weights $\mathrm{W}_{\mathrm{h}}(\mathrm{t})$. The CMAC weight tables are modified by steepest descent method and shown in Eq.4. In the equation $\mathrm{g}(\mathrm{t})$ denotes the gradient that used to update weights, J denotes the error criterion. And they are expressed as Eq.5 and Eq.6. In Eq.5 a, b and c are the appropriate positive constants set by designer.

$$
\begin{aligned}
& W_{p, h}{ }^{\text {new }}(t)=W_{p, h}^{\text {old }}(t)-g(t) \frac{\partial J}{\partial K_{p}} \frac{1}{K} \\
& W_{i, h}^{\text {new }}(t)=W_{i, h}^{\text {old }}(t)-g(t) \frac{\partial J}{\partial K_{i}} \frac{1}{K} \\
& W_{d, h}^{\text {new }}(t)=W_{d, h}^{\text {old }}(t)-g(t) \frac{\partial J}{\partial K_{d}} \frac{1}{K}
\end{aligned}
$$




$$
\begin{gathered}
g(t)=\frac{1}{c+a * \exp \left(-b\left|u^{*}(t)-u(t)\right|\right)} \\
J:=\frac{1}{2} \varepsilon(t)^{2} \\
\varepsilon(t)=u^{*}(t)-u(t)
\end{gathered}
$$

Moreover, the partial differential of Eq. (4) is developed as follows:

$$
\begin{aligned}
& \frac{\partial J}{\partial K_{p}}=\frac{\partial J}{\partial \varepsilon(t)} \frac{\partial \varepsilon(t)}{\partial u(t)} \frac{\partial u(t)}{\partial K_{p}} \\
& =\varepsilon(t)(y(t)-y(t-1)) \\
& \frac{\partial J}{\partial K_{i}}=\frac{\partial J}{\partial \varepsilon(t)} \frac{\partial \varepsilon(t)}{\partial u(t)} \frac{\partial u(t)}{\partial K_{i}} \\
& =-\varepsilon(t) e(t) \\
& \frac{\partial J}{\partial K_{d}}=\frac{\partial J}{\partial \varepsilon(t)} \frac{\partial \varepsilon(t)}{\partial u(t)} \frac{\partial u(t)}{\partial K_{d}} \\
& =\varepsilon(t)(y(t)-2 y(t-1)+y(t-2))
\end{aligned}
$$

\section{Experimental Evaluation}

\subsection{Experimental Equipment}

In order to illustrate the effectiveness of the proposed method, an experiment has been performed by using a beam and ball control model. The experiment equipment is shown in Fig.4. As the figure shows, there is an input manipulator for human to operate. It is set to record the data of human skill. Also there is a distance sensor on beam to read output data of the system. Moreover there is an Arduino board to transfer data between computer and system. The ball on the beam is the target to be controlled.

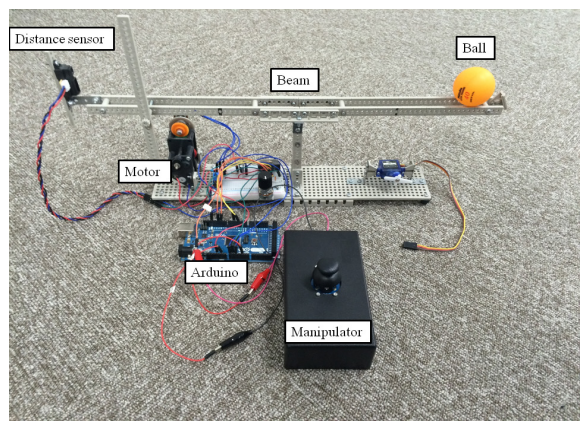

Fig. 4. Beam and Ball control Equipment

\subsection{Simulation and Experiment Result}

Aim of the experiment is to control the ball staying at the middle of the beam, where $r=0$. Position of ball will be considered as output $\mathrm{y}$ and in this experiment the initial $y$ is 15 , it means at the beginning the ball stays at the right side of the beam. Angle of the beam is considered as the input of the system and the initial input is 0 . The limit of $u$ is set as:

$$
-45^{\circ} \leq-u(t) \leq 45^{\circ}
$$

The output $\mathrm{y}$ is limited as:

$$
-15[\mathrm{~cm}] \leq y(t) \leq 15[\mathrm{~cm}]
$$

Human skill data $\mathrm{u}^{*}$ and $\mathrm{y}^{*}$ are obtained by skilled operator and they are shown in Fig.5. Based on the human skill data the learning process of CMACs is performed. In this design the CMACs with 4 weight tables and 3 labels are chosen. The coefficients of learning ratio are set to be $\mathrm{a}=10^{2}, \mathrm{~b}=10^{2}, \mathrm{c}=10^{2}$. In addition, the initial values of the weights are PID gains obtained by pole placement method based on the model of system. These PID gains are $\mathrm{K}_{\mathrm{p}}=0.0056, \mathrm{~K}_{\mathrm{i}}=$ $0.0019, \mathrm{~K}_{\mathrm{d}}=0.0045$.

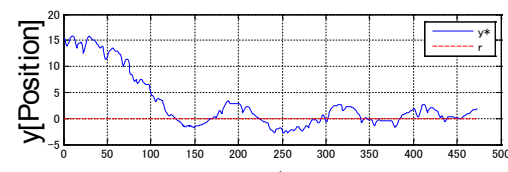
$\mathrm{t}$

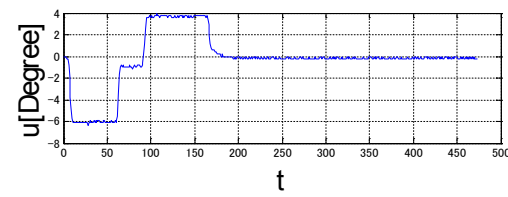

Fig.5. Control result by skilled operator

The learning process is evaluated by the following integrated squared error (ISE) equation:

$$
I S E=\sum_{t=1}^{6}\left(u^{*}(t)-u(t)\right)
$$

As Fig.6 shows, the learning process stops when the integrated squared error is less than 0.00051 .

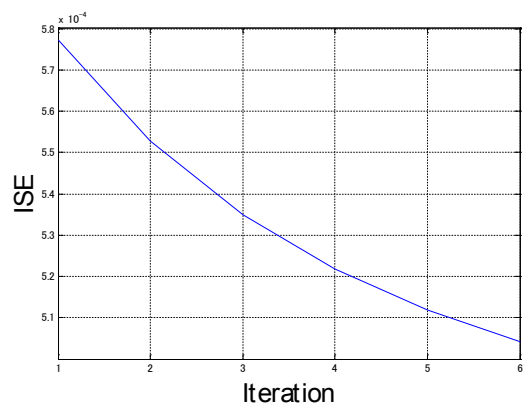

Fig.6. Performance of learning process

Fig.7 shows the comparison between learning result $u$ and teacher signal $\mathrm{u}^{*}$, where 6 iterations were performed. Fig. 8 shows the PID gains trajectories corresponding to Fig.7. By using the PID gains that shown in Fig.8 an experiment has been done and the result is shown in 
Fig.9.From figure $\mathrm{y}$ is close to $\mathrm{y}^{*}$ shows that by using proposed method human skill can be learned.

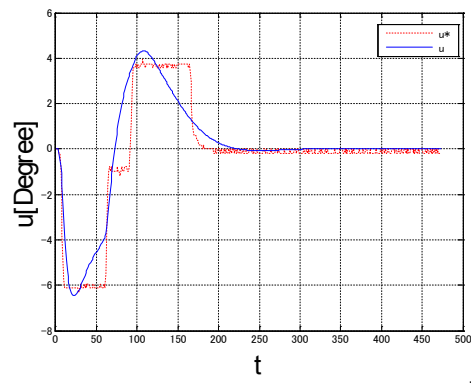

Fig.7. Comparison between $u$ and $\mathrm{u}^{*}$
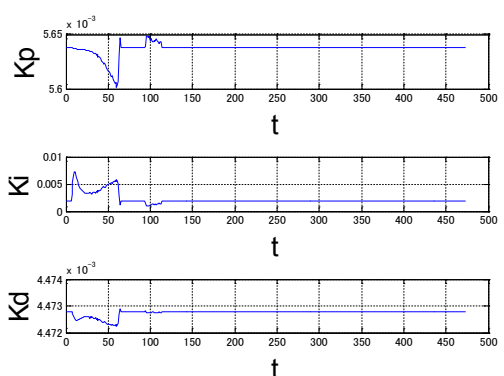

Fig.8.PID gains corresponding to Fig.7
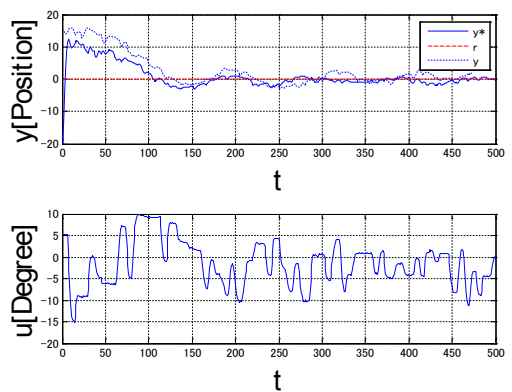

Fig.9. Control result by proposed method

\subsection{Human Skill Analysis}

Fig.10 shows the trajectories of $\mathrm{kc}$, Ti and $\mathrm{Td}$ they are got by following equations:

$$
k c=K p \quad T i=\frac{k c}{K i} T s \quad T d=\frac{K d}{k c} T s
$$

where Ts is sampling time and $T s=0.02$. From the figure human skill is analyzed as follows: At first $\mathrm{kc}$ and $\mathrm{Ti}$ become smaller and $\mathrm{Td}$ becomes bigger because manipulator tries to move the ball to target stably. Then $\mathrm{kc}$ and $\mathrm{Ti}$ becomes bigger and $\mathrm{Td}$ becomes smaller shows human thinks the ball is going to reach the target and he is trying to stop the ball.

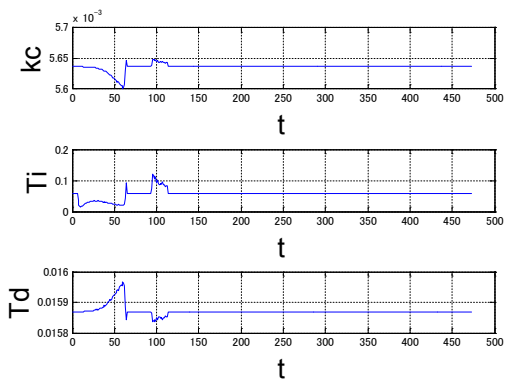

Fig.10. Trajectories of PID parameters

Then $\mathrm{kc}$ and Ti becomes bigger and Td becomes smaller because the manipulator tries to stop the ball again. At last, $\mathrm{kc}$ and $\mathrm{Ti}$ become smaller and $\mathrm{Td}$ becomes bigger to stop the ball at the target after that there is no change of $\mathrm{kc}, \mathrm{Ti}$ and $\mathrm{Td}$ shows human thinks the ball stops at the target. From analysis, the manipulator wants to control the ball stably approach to the target without overshoot.

\section{Conclusions}

In this paper, a human skill based PID controller using $\mathrm{CMAC}$ has been proposed. And the effectiveness of method has been experimentally examined on a beam and ball model. According to the control results the proposed method can replace human skill and based on the PID trajectories human skill can be analyzed.

\section{References}

1. S.Liu and H.Asada "Adaptive Control of Deburring Robots Based on Human Skill Models," Proc.of IEEE Conf. on Decision and Con- trol,Brighton, pp.348 353,1991 .

2. K.S.Narendra and K.Parthasarathy "Identification and Control of Dy- namical Systems Using Neural Networks," IEEE Trans.on Neural Networks, Vol.1,No.1,pp.4-27,1990.

3. S.Omatu, K.Marzuki and Y.Rubiyah "Neuro-Control and Its Applica- tions," Springer-Verlag, 1995.

4. M.Kato, T.Yamamoto and S.Fujisawa "A Skill-Based PID Controller Using Artificial Neural Networks," Proc.of Int. Conf. on Computational Intelligence for Modeling, Control and Automation, Vienna, pp.702705,2005 .

5. K.Koiwai, K.Kawada and T.Yamamoto, "Design of a Human-Skill Based PID Controller using CMACs," Int. Conf. on Control, Automation and Systems, 2008 